\title{
Comparative Role of Sonourethrography (SUG) and Magnetic Resonance Urethrography (MRU) in Anterior Male Urethral Strictures
}

\author{
Rajul Rastogi, Pawan Joon, Arawat Pushkarna, Arjit Agarwal, Asif Majid Wani, Prabhat Kumar \\ Bhagat, Yuktika Gupta, Shourya Sharma, Pankaj Kumar Das, Sagar Parashar, Pragya Sinha, Mohini \\ Chaudhary and Vijai Pratap
}

Department of Radiodiagnosis and Urology, Teerthanker Mahaveer Medical College and Research Center, Teerthanker Mahaveer University, Moradabad, UP, India

Corresponding author: Rajul Rastogi, Department of Radiodiagnosis and Urology, Teerthanker Mahaveer Medical College and Research Center, Teerthanker Mahaveer University, Moradabad, UP, India, Tel: 919319942162; E-mail: eesharastogi@gmail.com

Received: 02 December 2016; Accepted: 22 December 2016; Published: 27 December 2016

Citation: Rastogi R, Joon P, Pushkarna A, et al. Comparative Role of Sonourethrography (SUG) and Magnetic Resonance Urethrography (MRU) in Anterior Male Urethral Strictures. Ann Clin Lab Res. 2016, 4: 4.

\section{Abstract}

Anterior urethral stricture is a common clinical condition encountered in day-to-day urological practice affecting predominantly males. Retrograde urethrography (RGU) is a common investigation done to determine the future course of management. But with increasing use of plastic surgery and other endoscopic procedures, information regarding presence of spongiofibrosis and other associated conditions, in addition to length of stricture is being demanded from radiologist for deciding the mode of management. Sonourethrography (SUG) has started gaining acceptance over RGU in last few decades for determining periurethral spongiofibrosis which at the same time obviates radiation to gonads. In recent years, magnetic resonance urethrography (MRU) has also been advocated for evaluation of anterior urethral strictures due to its excellent soft tissue contrast and lack of operator dependence. Besides, MRU can delineate posterior urethra simultaneously, affecting further management obviating the need for micturating cystourethrogram (MCU). Hence, in this article we evaluate the comparative role of SUG ad MRU in the evaluation of male anterior urethral strictures.

Keywords: Urethra, Stricture, Sonourethrography, Magnetic resonance, Urethrography, Spongiofibrosis

\section{Introduction}

Strictures or luminal narrowing of male urethra are broadly categorized in to anterior and posterior type depending upon location with former being predominantly secondary to straddle injury, iatrogenic urethral catheterization, urethritis (infection or inflammation) and balanitis xerotica obliterans while latter is usually secondary to injury.

Anterior urethral stricture (AUS) may be associated with periurethral fibrosis also known as spongiofibrosis while posterior urethral stricture (PUS) may be associated with loss of urethral axis in traumatic cases, information very necessary for optimal management.

Both SUG and MRU have been found to be superior to RGU in evaluation and prediction of the mode of management of AUS which prompted us to conduct this study of comparing the role of SUG and MRU.

\section{Materials and Methods}

Twenty male patients with first-time diagnosis of anterior urethral stricture on RGU were referred to our department for MRU that was performed on 1.5 Tesla MRI scanner following distension of the urethra by instillation of optimal amounts of sterile gel per urethram and application of soft-plastic, peniletip clamp. High-resolution, T2 weighted images were obtained in all planes (Figure 1). SUG was performed immediately following $\mathrm{MRU}$ with a high-resolution, linear-array, $10-14 \mathrm{MHz}$ transducer through penile and transperineal technique (Figure 2). Data related to site and length of stricture; presence or absence of spongiofibrosis with its extent and any other associated abnormality was recorded in both SUG and MRU. 


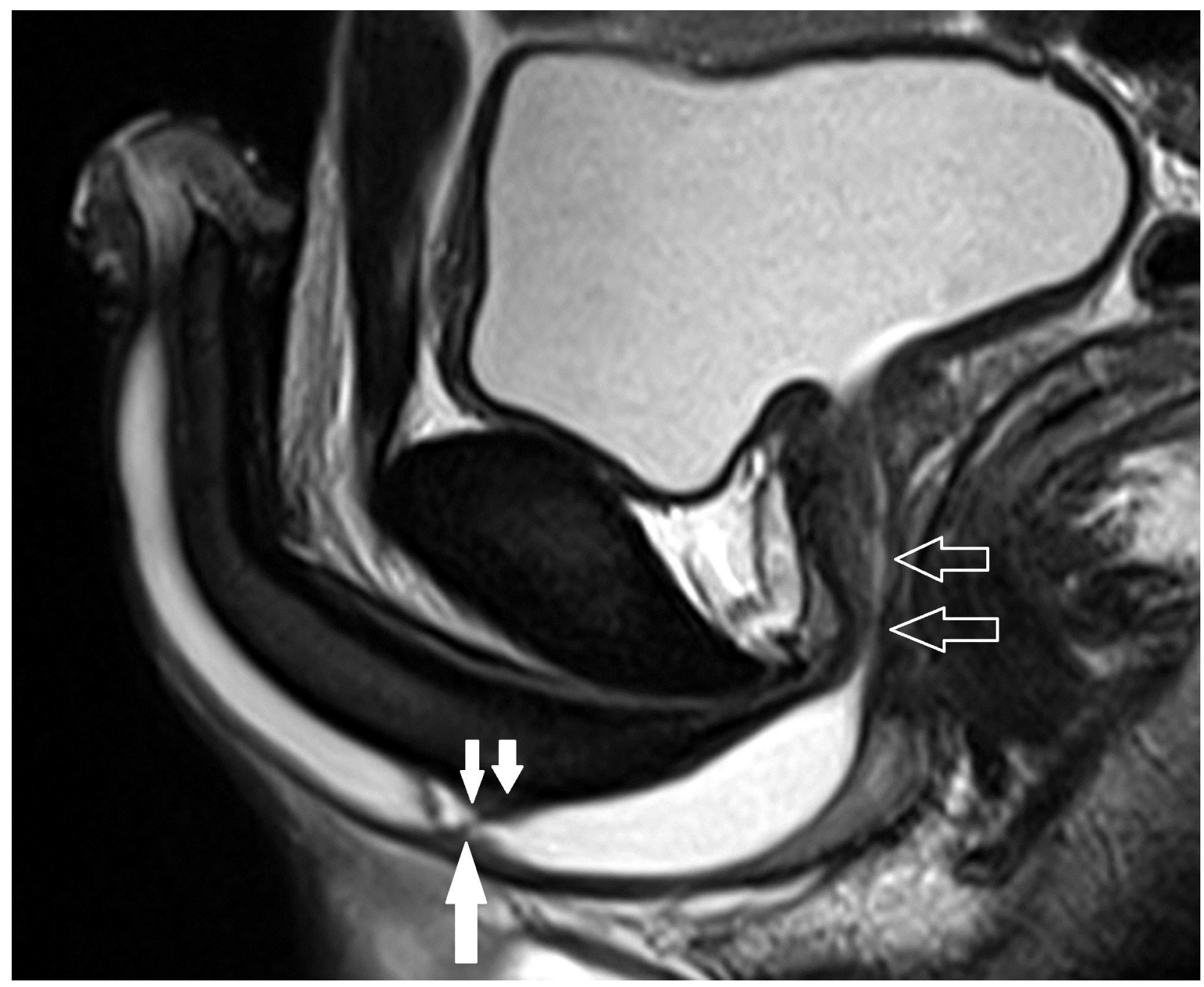

Figure 1 T2 weighted, sagittal, MR image in a patient shows anterior urethral stricture (large white solid arrow) and spongiofibrosis (small white solid arrows) with normal-appearing posterior urethra (hollow arrows).

An experienced radiologist blinded to both RGU and MRU findings, recorded the SUG findings. While the radiologist recorded MRU findings, he was blinded about the RGU and SUG findings of the patient.

\section{Observations and analysis}

Out of 20 patients, three patients were excluded from our study due to suboptimal quality of MRI scan.

The age of the patients ranged from 18 to 72 years with 10 patients having history of infection, 3 with history of trauma, 3 with history of previous urethral instrumentation and one with none of the above histories i.e. idiopathic.

Long-segment stricture in anterior urethra was detected in 10 out of 17 (58.8\%) patients by SUG while MRU detected it in 14 out of $17(82.4 \%)$ patients meaning thereby that 4 patients were falsely-diagnosed as short-segment stricture by SUG out of which two were in bulbar and two in penobulbar urethra. Thus, SUG has a sensitivity, specificity, and accuracy of $71.4 \%$, $42.9 \%$ and $76.5 \%$ respectively.
Out of 17 patients, 12 patients revealed spongiofibrosis on both SUG and MRU while 5 had no signs of spongiofibrosis. Out of 4 patients that were falsely-negative for long-segment stricture on SUG, 3 had spongiofibrosis and one did not have any signs of spongiofibrosis.

Out of 17 patients, one patient had anterior urethral diverticulum and one had posterior urethral calculus with small sinus in to periurethral tissues. Though, urethral diverticulum was detected on both SUG and MRU yet it was better delineated on the latter. Posterior urethral calculus with sinus was well delineated only on MRU.

Three out of 17 patients had concomitant posterior urethral stricture that were revealed on MRU but not detected by SUG. All these three patients had long-segment anterior urethral strictures. Distraction of posterior urethra was well visualized in one of these three cases only on MRU which was secondary to trauma, obviating the need for micturating cystourethrogram.

Based on the MRU findings, open reconstructive surgery of urethra was planned in 17 out of 17 cases due to presence of 
either long-segment stricture; presence of spongiofibrosis, urethral diverticulum and periurethral sinus or stricture in the posterior urethra with urethral calculus. Based on the SUG findings, however, 16 out of 17 patients could have been planned for open reconstructive surgery of urethra yielding an accuracy of $94.1 \%$ when compared with MRU. The patient where the MRU made a significant difference in decision making was the one false-diagnosed as short-segment stricture on SUG in the absence of any other associated finding.

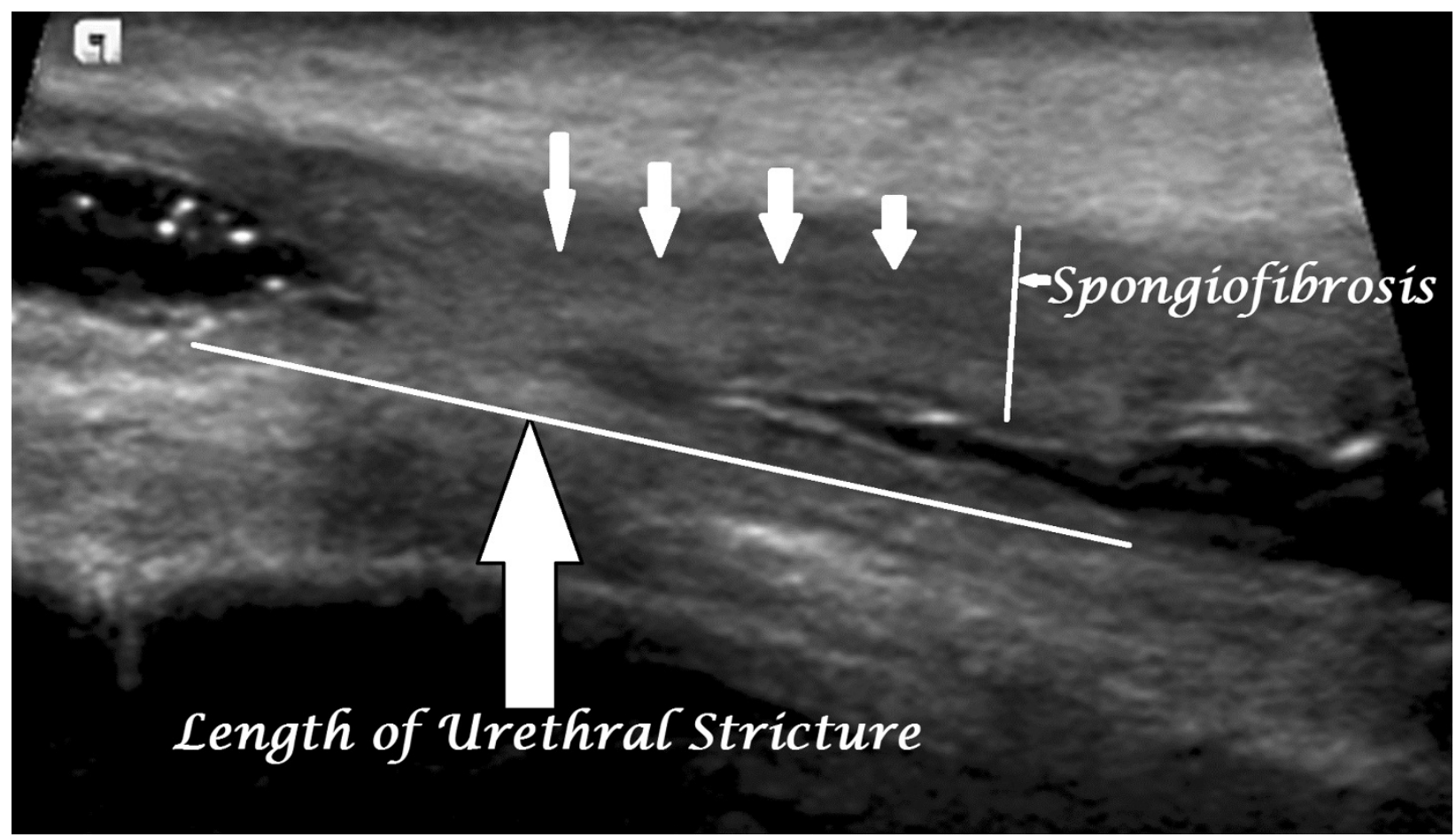

Figure 2 SUG image shows anterior urethral stricture associated with spongiofibrosis.

\section{Discussion}

Management of male anterior urethral strictures depends upon multiple factors including length of urethral stricture (short segment stricture is defined as a stricture less than or equal to $1.5 \mathrm{~cm}$ in length); presence or absence of spongiofibrosis; alterations in posterior urethral axis and other significant associated findings especially urethral diverticulum, sinus and calculus [1].

For reducing recurrence rates, long-segment strictures and strictures with associated spongiofibrosis, diverticulum and periurethral sinus are treated with open reconstructive surgery while isolated short-segment strictures usually respond well to simple dilatation or endoscopic internal urethrotomy $[2,3]$.

Though RGU has been the mainstay of evaluating male anterior urethral strictures for several decades yet its inherent disadvantages of radiation-exposure and need for high technical expertise paved the way better imaging modality like SUG and MRU $[4,5]$.

Sonography due to its inherent advantage of being radiation-free was first used in evaluation of male urethral strictures in last decade of 20th century [6]. SUG is superior to RGU in demonstrating presence of spongiofibrosis [6,7]. However, its major limitation is operator dependence; smaller field of view and suboptimal or no information about posterior urethra, urethral axis and periurethral sinus/fistula. Recently, use of sonoelastography has been suggested to be superior to RGU and SUG in estimating the length of urethral stricture and degree of spongiofibrosis [8].

Like SUG, MRU is also highly accurate in determining the location and length of male anterior urethral strictures and associated spongiofibrosis $[1,6,9]$. However, unlike SUG, MRU offers higher field of view allowing simultaneous visualization of posterior urethra with its axis in distracting injuries as well as associated conditions that might affect the final management as urethral diverticulum, urethral calculus/tumor and periurethral sinus/fistula more conspicuously [6]. In spite of being so informative and reproducible MRU has limited availability and expertise; is expensive; has higher examination time; has higher incidence of claustrophobia and is absolutely contraindicated in patients with cochlear implants and nonMRI compatible pacemaker.

Very few studies similar to ours, comparing SUG and MRU have been described in the medical literature. El-Ghar et al. revealed that MRU has a higher sensitivity, specificity and accuracy than that of SUG in evaluating male urethral strictures as in our study [6]. Their study also revealed that sensitivity, specificity and accuracy similar to that of MRU can be achieved by a combination of RGU and SUG. 


\section{Limitations of the Study}

Small sample size may alter the results

\section{Conclusion}

MRU is superior to SUG in detecting the length of male anterior urethral strictures.

SUG has limited accuracy in evaluating penobulbar and bulbar urethral strictures.

SUG accuracy is similar to MRU in detection of spongiofibrosis.

SUG has limited ability to evaluate concomitant urethral diverticulum and periurethral sinus fistula in cases of male anterior urethral strictures.

SUG has no significant role in detection/evaluation of posterior urethral stricture or urethral axis that may coexist.

SUG is similar to MRU in its ability to guide the mode of management of male anterior urethral strictures.

\section{Summary}

To summarize, though SUG is highly accurate in detecting spongiofibrosis and in deciding the mode of management yet it has limited ability in evaluating the length of male anterior urethral strictures and associated features like urethral diverticulum or periurethral sinus/fistula. MRU can serve as a single-stop shop providing all necessary information about anterior and posterior urethra needed for best management of anterior male urethral strictures. However, the decision of using SUG or MRU should not only be based on clinical condition and time, technique and expertise availability but also financial viability.

\section{References}

1. Osman Y, Abou ELghar M, Mansour O (2006) Magnetic resonance urethrography in comparison to retrograde urethrography in diagnosis of male urethral stricture: is it clinically relevant? Eur Urol 50: 587-594.

2. MacDonald MF, Santucci RA (2005) Review and treatment algorithm of open surgical techniques for management of urethral strictures. Urology 65: 9-45.

3. El-Bab TKF, Abdelhamid AM, Galal EM, Amin MF (2013) Magnetic resonance urethrography versus conventional retrograde urethrography for diagnosis of anterior urethral stricture. J Am Sci 9: 238-242.

4. Sakti D (1992) Ultrasonographic evaluation of the urethral stricture disease. Urology 40: 237-242.

5. Narumi $Y$, Hricak $H$, Armenakas $N$ (1993) MR imaging of traumatic posterior urethral injury. Radiology 188: 439-443.

6. El-Ghar MA, Osman Y, Elbaz ES, Refiae H, El-Diasty T (2010) MR urethrogram versus combined retrograde urethrogram and sonourethrography in diagnosis of urethral stricture. Eur J Radiol 74: 193-198.

7. Mitterberger M, Christian G, Pinggera GM (2007) Gray scale and color Doppler sonography with extended field of view technique for the diagnostic evaluation of anterior urethral strictures. J Urol 177: 992-997.

8. Talreja SM, Tomar V, Yadav SS, Jaipal U, Priyadarshi S, et al. (2016) Comparison of sonoelastography with sonourethrography and retrograde urethrography in the evaluation of male anterior urethral strictures. Turk J Urol 42: 84-91.

9. Sung DJ, Kim YH, Cho SB (2006) Obliterative urethral stricture: MR Urethrography versus conventional retrograde urethrography with voiding Cystourethrography. Radiology 240: 842-848. 